\title{
Mensurando a Credibilidade do Regime de Metas Inflacionárias no Brasil
}

\author{
Measuring the credibility of the inflation targeting in Brazil
}

HELDER FERREIRA DE MENDONÇA****

\begin{abstract}
RESUMO: O objetivo deste artigo é aplicar dois índices para medir a credibilidade da meta de inflação no Brasil e analisar seus resultados. Os resultados indicam que a estrutura adotada para este regime monetário no Brasil não é adequada para construir credibilidade. Palavras-chave: Credibilidade; metas de inflação; expectativa de inflação.
\end{abstract}

ABSTRACT: The aim of this paper is to apply two indexes for measuring the credibility of the inflation targeting in Brazil and to analyze their outcomes. The findings denote that the framework adopted for this monetary regime in Brazil is not adequate for building credibility.

KEYWORDS: Credibility; inflation targets; inflation expectation.

JEL Classification: E31; E52.

\section{INTRODUÇÃO}

Desde as contribuições de Kydland e Prescott (1977) e de Barro e Gordon (1983) à literatura econômica, a análise sobre a credibilidade da política econômica tornou-se um importante objeto de estudo para os economistas. Ao longo da década de 1990 o regime de metas de inflação tornou-se um instrumento utilizado por diversos países como forma de institucionalizar o objetivo prioritário da busca da estabilidade de preços e dar maior transparência à condução da política monetária. Em outras palavras, o regime de metas de inflação representa uma estrutura que tem por finalidade eliminar os problemas relacionados à inconsistência dinâmica, e por conseguinte, conquistar credibilidade para a autoridade monetária.

Para que se desenvolva a credibilidade é preciso, em um primeiro momento,

\footnotetext{
* Professor do Departamento de Economia da Universidade Federal Fluminense - UFF, Niterói, RJ, Brasil. E-mail: helderfm@hotmail.com. ISubmetido: novembro 2002; aceito: junho 2003.

* O autor é pesquisador do CNPq.
} 
que o responsável pela condução da política econômica conquiste reputação. Para tanto, é necessário que os agentes econômicos acreditem que uma política anunciada para um determinado instante no tempo seja implementada com êxito. Ou seja, o anúncio de uma política econômica a ser colocada em prática contará com credibilidade se o público espera uma chance reduzida da ocorrência de inconsistência temporal. Logo, se por exemplo, o Banco Central teve sucesso na obtenção das metas de inflação ao longo do tempo (o que implica conquista de reputação) os agentes econômicos acreditam que a autoridade monetária terá sucesso no alcance da meta futura, o que por conseguinte, implica credibilidade elevada. ${ }^{1}$

Uma alta credibilidade operacional do regime de metas de inflação é conseqüência da demonstração de competência do Banco Central na condução da política monetária em busca da meta anunciada. (Andersson e Berg, 1995) Sob essa perspectiva, tendo transcorrido alguns anos desde a introdução da estratégia de metas de inflação em vários países, a mensuração da credibilidade deste regime monetário tem despertado o interesse de diversos economistas para a elaboração de índices com essa finalidade.

O objetivo deste artigo é fazer uma aplicação de dois índices para mensurar a credibilidade do regime de metas de inflação adotado no Brasil e efetuar a análise dos resultados obtidos.

\section{MENSURANDO A CREDIBILIDADE DO BCB}

Em recente artigo para o proceedings da XXVI Conferência Anual de Política Econômica do Federal Reserve Bank of St. Louis, Cecchetti e Krause (2002) apresentaram um índice de credibilidade para o Banco Central (ICCK) partindo da definição de credibilidade feita por Cukierman e Meltzer (1986, p. 1108 ) "the absolute value of the difference between the policymaker's plans and the public's beliefs about those plans". Nesse sentido, tal como fora proposto por Svensson (2000), a credibilidade pode ser medida pela diferença entre a inflação esperada e a meta:

(1) $I C_{C K}=\left\{\begin{array}{lll}1 & \text { se } & E(\pi) \leq \pi_{t} \\ 1-\frac{1}{0,2-\pi_{t}}\left[E(\pi)-\pi_{t}\right] & \text { se } & \pi_{t}<E(\pi)<20 \% \\ 0 & \text { se } & E(\pi) \geq 20 \%\end{array}\right\}$

O índice de credibilidade assume o valor 1 se a inflação anual esperada é menor ou igual à meta de inflação $(\pi t)$ e decresce de forma linear à medida que

\footnotetext{
${ }^{1}$ Para uma análise sobre a teoria da credibilidade da política monetária, ver de Mendonça (2002).
} 
a inflação esperada aumenta. No caso de a inflação esperada ser superior a $20 \%$ é admitido que o índice tem valor igual a zero. Portanto, o índice varia entre zero (credibilidade nula) e 1 (credibilidade máxima).

Com base na metodologia supracitada foi medida a credibilidade para o regime de metas de inflação implementado no Brasil a partir das informações disponibilizadas pelo Banco Central do Brasil sobre as expectativas do mercado (com início em janeiro de 2000) para a inflação e as respectivas metas anuais. Essas informações têm freqüência diária (semana de 5 dias), totalizando 740 observações até $1^{\circ}$ de novembro de 2002.

Deve-se ressaltar que o índice desenvolvido por Ceccketti e Krause (2002) foi construído com o objetivo de efetuar comparações internacionais. Assim, os autores mencionados utilizaram como meta para a inflação o que a literatura considera como meta padrão, isto é, $2 \%$. O limite de $20 \%$ introduzido na elaboração do índice significa que os autores consideram que uma vez ultrapassado esse nível o Banco Central perde o controle sobre a inflação. Com o objetivo de adaptar esse índice para o caso brasileiro, ao invés da meta de $2 \%$ foram consideradas as metas anuais de inflação definidas pelo Banco Central do Brasil para a obtenção do indicador. ${ }^{2}$ A evolução do índice ao longo do tempo pode ser observada por meio da figura 1 .

Figura 1: Evolução da credibilidade (IC Cecchetti e Krause)

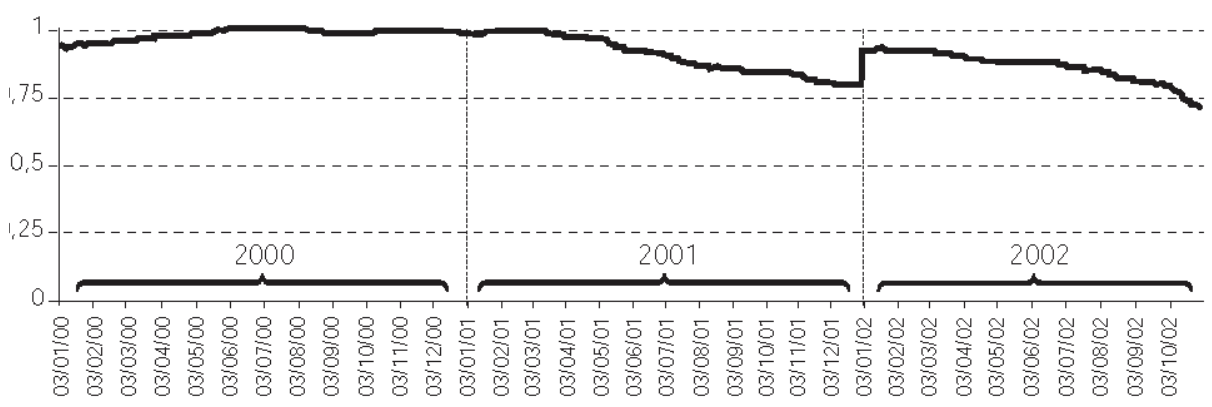

De acordo com a figura 1 observa-se que a credibilidade manteve-se elevada ao longo dos três anos em consideração (IC>0,7). Apesar disso, observa-se um padrão de comportamento distinto para cada ano. No ano 2000 a credibilidade sustentou-se em um patamar muito elevado e relativamente estável durante todo o ano. Para o ano 2001 verifica-se que o nível de credibilidade também foi elevado, mas apresentou decréscimo até atingir um nível aproxi-

\footnotetext{
22 Para o ano 2000 a meta foi fixada em $6 \%$ com intervalo de tolerância de $\pm 2 \%$; para o ano 2001 a meta foi fixada em $4 \%$ com intervalo de $\pm 2 \%$; e em 2002 a meta corresponde a $3,5 \%$ com intervalo de $\pm 2 \%$.
} 
madamente $20 \%$ abaixo do ano anterior. Em 2002 o índice de credibilidade apresentou uma performance inferior à dos anos anteriores. Diante desses resultados, pode-se dizer que a credibilidade do regime de metas de inflação tem se encontrado em um nível elevado. Contudo, os sinais de redução no índice sugerem a necessidade de cuidados para evitar uma acentuada perda de credibilidade.

Recentemente, Sicsú (2002) desenvolveu um índice de credibilidade específico para o regime de metas de inflação introduzido no Brasil, cuja essência pode ser compreendida da seguinte forma:

Se um objetivo de política econômica é crível, isto significa que o mercado acredita que pode ser alcançado. Então, uma meta de inflação para um determinado período é plenamente crível se é igual à expectativa de inflação do mercado para o mesmo período, sendo o contrário verdadeiro: se a expectativa de inflação do mercado está bastante distante da meta de inflação do banco central, isto significa que tal objetivo de política econômica carece de credibilidade. (Sicsú, 2002, p. 3)

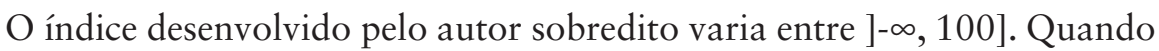
o índice se aproxima de 100 significa que o mercado tem certeza de que a meta central será obtida. Por outro lado, quando o índice torna-se negativo, o mercado espera que os limites para a flutuação da inflação não sejam respeitados, e por conseguinte, o mercado está convencido de que a meta não será obtida.

Apesar da idéia acima não estar incorreta, o limite inferior para o índice mostra-se inadequado para ser aplicado em análises futuras envolvendo variáveis macroeconômicas. Com objetivo de eliminar a imprecisão mencionada e atender à sugestão feita por Svensson (2000), foi elaborado um índice de credibilidade (normalizado entre 0 e 1 ) que contempla os desvios da inflação esperada em relação à meta de inflação. Logo,

$$
\text { (2) } I C=\left\{\begin{array}{ll}
1 & \text { se } E(\pi)=\pi_{t} \\
1-\frac{1}{\pi_{t}^{*}-\pi_{t}}\left[E(\pi)-\pi_{t}\right] & \text { se } \pi_{t M I N}^{*}<E(\pi)<\pi_{t M A X}^{*} \\
\text { se } E(\pi) \geq \pi_{t M A X}^{*} \text { ou } E(\pi) \leq \pi_{t M I N}^{*}
\end{array}\right\}
$$

A implementação do regime de metas de inflação no Brasil, em junho de 1999, tem como principal característica a adoção de uma meta central para inflação (variação do IPCA) e a possibilidade de desvios de dois pontos percentuais para cima ou para baixo. ${ }^{3}$ Com o objetivo de apreender a estratégia adotada, o índice de credibilidade possui valor igual a 1 quando a inflação anual esperada $(E(\pi))$ é igual à meta central e decresce de forma linear à medida que

\footnotetext{
${ }^{3}$ Para uma análise sobre o regime de metas de inflação adotado no Brasil, ver de Mendonça (2001).
} 
a expectativa inflacionária se desvia da meta anunciada. Destarte, o índice de credibilidade apresenta valor entre zero e 1 se a inflação esperada estiver dentro dos limites máximo e mínimo $\left(\pi^{*}\right)$ estabelecidos para cada ano e assume valor zero quando a inflação esperada ultrapassa um desses limites. Com base nessa metodologia foi mensurada a credibilidade para as metas inflacionárias levando em conta as informações disponibilizadas pelo Banco Central do Brasil e que foram utilizadas na elaboração do índice anterior. A evolução do índice pode ser observada na figura 2.

De acordo com este índice observa-se que a credibilidade apresentou um bom desempenho no ano 2000. Logo no início do ano (março) o índice ultrapassou o nível de 0,75 e continuou se elevando até que, a partir do início do mês de maio, mostrou-se estável, variando entre 0,85 e 1 . Porém, a mesma performance não é observada para os anos subseqüentes. O êxito no ano $2000 \mathrm{fez}$ com que o início do ano 2001 fosse marcado por uma elevada confiança do mercado na obtenção da meta anunciada. Não obstante, a alta credibilidade erodiu-se rapidamente. A justificativa para que o índice se tornasse nulo a partir de 23 de julho foi a conjunção de três elementos básicos: o anúncio do racionamento de energia elétrica, a crise que se anunciava na Argentina e a queda na atividade econômica mundial.

Figura 2: Evolução da credibilidade

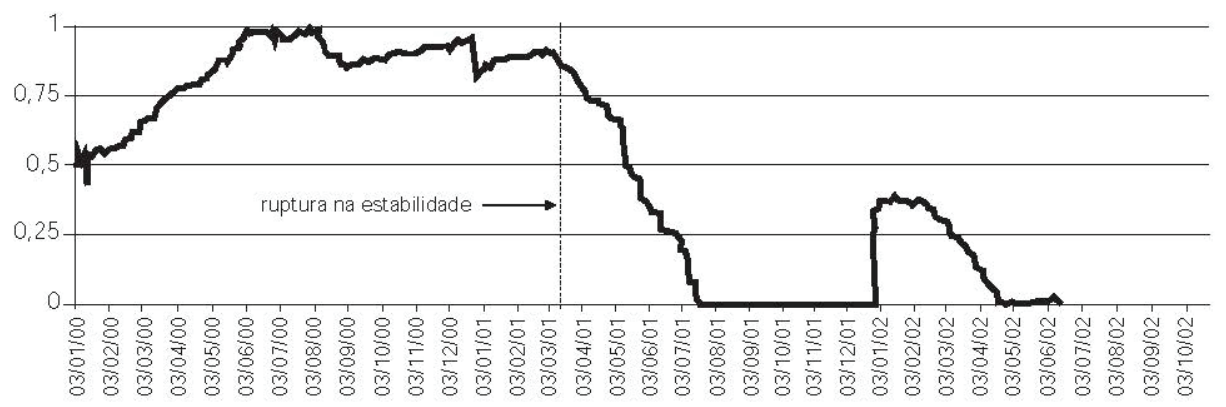

O índice de credibilidade no ano 2002, conforme pode ser observado na figura 2, em nenhum momento apresentou um nível satisfatório. Ainda que o início de cada período (ano) implique uma nova possibilidade para a obtenção da meta anunciada, o insucesso verificado no ano de $2001 \mathrm{fez}$ com que o mercado iniciasse o ano com uma expectativa inferior a 39\% das chances do Banco Central do Brasil alcançar a meta de 3,5\%. No começo de abril a credibilidade encontrava-se muito próxima de zero e tornou-se nula a partir de junho. A instabilidade na bolsa americana, os prejuízos em bônus corporativos de empresas dos EUA e o histórico recente da crise argentina afugentaram os investidores dos títulos dos países emergentes fazendo com que o risco do país aumentasse de forma significativa. Como conseqüência, houve uma grande 
demanda dos investidores por dólares para que enviassem recursos para fora do país. Um outro elemento que não deveser esquecido é a especulação que se manifestou durante o período das eleições presidenciais. $\mathrm{O}$ resultado desses fatores adversos foi uma acentuada desvalorização cambial que foi decisiva para o niilismo do mercado no alcance da meta de inflação.

\section{CONSIDERAÇÕES FINAIS}

A seção anterior revela um resultado paradoxal quando são comparados os dois índices. Enquanto o índice Cecchetti e Crause revela um elevado nível de credibilidade para o período recente, o outro índice de credibilidade revela um baixíssimo nível. O motivo para a dissensão encontrada se deve à metodologia utilizada para a elaboração dos índices. No primeiro caso, foi levada em conta a meta ideal para inflação $(2 \%)$, mas para que haja perda total de credibilidade é preciso que a inflação ultrapasse a taxa de $20 \%$. No segundo índice, a meta de inflação utilizada foi maior que $2 \%$, entretanto, o limite de flutuação de $2 \%$ para cima ou para baixo em relação à meta central mostrou-se muito rigoroso para a obtenção de credibilidade.

A instalação do regime de metas para a inflação no Brasil tinha como objetivo precípuo fazer com que a inflação proveniente do ataque especulativo contra o Real fosse eliminada de forma gradativa. Diante dessa tarefa, o então Ministro da Fazenda e o Presidente do Banco Central tomaram a decisão de que as metas não deveriam contar com cláusulas de escape, pois o Banco Central do Brasil deveria demonstrar competência no alcance das metas anunciadas (independente da incidência de choques) para que pudesse ser desenvolvida a credibilidade. ${ }^{4}$ Conforme foi exposto pelo segundo índice analisado, esta estrutura não se mostrou adequada para os objetivos pretendidos.

Os resultados encontrados indicam que a estratégia de metas de inflação, tal como foi introduzida no Brasil (sem cláusulas de escape), não se mostrou capaz de se sustentar diante de choques de oferta e da volatilidade da taxa de câmbio. Nesse sentido, é importante que esta âncora nominal sofra as devidas alterações de forma que o Banco Central do Brasil recupere sua reputação no controle da inflação para que possa desenvolver credibilidade. A estrutura na qual está baseado o primeiro índice analisado mostra-se mais flexível e pode ajudar no alcance desse objetivo, pois a utilização da meta de inflação ideal $(2 \%)$ combinada a limite de $20 \%$ permite maior possibilidade para o Banco Central construir credibilidade.

\footnotetext{
${ }^{4}$ A possibilidade do uso de cláusulas de escape em situações extremas implica que não haja perda de credibilidade quando a meta não é obtida, pois a mudança na política planejada não é resultado da adoção de políticas inconsistentes no tempo, mas resultado de variáveis que não podem ser mensuradas.
} 
É diante do risco de haver uma perda de credibilidade definitiva na estratégia de metas de inflação adotada no Brasil que o Banco Central decidiu fazer alterações na meta e na banda de flutuação da inflação para os anos de 2003 e 2004. A meta central de inflação de 2003 alterou-se de 3,25\% para $4 \%$ e a de 2004 foi fixada em $3,75 \%$. Além dessas mudanças, a margem de erro para o alcance das metas foi alterada de $2 \%$ (para cima ou para baixo) para 2,5\%. Essas mudanças sugerem que o Banco Central do Brasil decidiu adotar uma estratégia gradualista para ajustar o regime de inflação. Entretanto, tais alterações podem se mostrar insuficientes para os próximos anos.

Um problema a ser corrigido para o atual regime de metas para a inflação no Brasil refere-se ao horizonte de tempo para a definição das metas. A ousadia do Banco Central do Brasil em definir as metas de inflação para vários anos representa um risco para a própria sobrevivência deste tipo de âncora nominal. Economias que são muito sensíveis a choques externos, tal como é a economia brasileira, e que por conseguinte, são muito instáveis, não permitem um planejamento razoável para o comportamento futuro da inflação. Sendo assim, para que haja uma recuperação da credibilidade neste regime monetário e que o objetivo de servir como um guia para a formação das expectativas dos agentes econômicos seja alcançado, não é preciso anunciar metas inflacionárias para vários anos. Um anúncio anual da meta de inflação em comum acordo entre o governo e o Banco Central do Brasil representaria um aumento da qualidade para a definição da meta, pois seria possível analisar as condições da economia e projetá-las para um período menor.

Conforme indicado pelo índice Cecchetti e Krause é possível a definição de uma meta para a inflação até um limite superior próximo a $20 \%$. É óbvio que este limite não representa uma taxa de inflação que se considere ideal, entretanto, ela representa o nível máximo em que o Banco Central pode controlá-la de forma efetiva. O ponto a ser considerado é que o uso do regime de metas de inflação requer não apenas a definição de uma meta desejável (2\%), mas uma avaliação das condições da economia para que seja definida a meta. É melhor a determinação de uma meta para a inflação elevada em cuja consecução o público acredite (tal como ocorreu em 1999)5 do que a definição de uma meta de inflação que não possui credibilidade.

A conseqüência de o Banco Central do Brasil tentar conseguir reputação de forma rápida via escolha de metas para a inflação abaixo do que realmente pode ser alcançado tende a ser desastrosa. Sob tais circunstâncias, a necessidade de um aumento da taxa de juros para fazer com que as expectativas convirjam para a meta anunciada tende a provocar uma combinação de elevação da dívida pública com desaceleração da atividade econômica que pode colocar em

\footnotetext{
${ }^{5}$ A meta de inflação definida para o ano de 1999 foi de $8 \%$ com intervalo de tolerância de $\pm 2 \%$.
} 
risco a estabilidade macroeconômica. Portanto, o problema da credibilidade do Banco Central não se concentra no regime utilizado, mas na forma como as metas são definidas em termos de sua magnitude e de seu horizonte temporal.

\section{REFERÊNCIAS BIBLIOGRÁFICAS}

ANDERSSON, K. e BERG, C. (1995) “The Inflation target in Sweden”. In: Haldane, A.G. (ed.) Targeting Inflation. Bank of England, 207-225.

BARRO, R. J. e GORDON, D. (1983) "Rules, Discretion and Reputation in a Model of Monetary Policy”. Journal of Monetary Economics, 12, North-Holland, 101-121.

CECCHETTI, S.G. e KRAUSE, S. (2002) "Central Bank Structure, Policy Efficiency, and Macroeconomic Perfomance: Exploring Empirical Relationships”. Review, Federal Reserve Bank of St. Louis, JulyAugust, 47-59.

CUKIERMAN, A. e MELTZER, A.H. (1986) "A Theory of Ambiguity, Credibility, and Inflation under Discretion and Asymmetric Information”. Econometrica, September, 54(5), 1099-128.

de MENDONÇA, H.F. (2002) “A Teoria da Credibilidade da Política Monetária: Desdobramentos do Debate Regras Versus Discrição”. Revista de Economia Política, vol. 22, no 3 (87), julho-setembro, 46-64.

. (2001) "Metas de inflação: uma análise preliminar para o caso brasileiro", Economia Aplicada, vol. 5, n $\mathrm{n}^{\mathrm{O}} 1$, janeiro-março, 129-158.

KYDLAND, F. E. e PRESCOTT, E. C. (1977) "Rules Rather than Discretion: the Inconsistency of Optimal Plans". Journal of Political Economic, vol. 85, n 3, 473-492.

SICSÚ, J. (2002) "Expectativas Inflacionárias no Regime de Metas de Inflação: uma análise preliminar do caso brasileiro". Economia Aplicada, vol. 6, n 4, 703-711.

SVENSSON, L. (2000) "How Should Monetary Policy Be Conducted in an Era of Price Stability?" NBER Working Paper Series, Cambridge, February. 